\title{
Electrically controllable plasmon induced reflectance in hybrid metamaterials
}

Cite as: Appl. Phys. Lett. 113, 221105 (2018); https://doi.org/10.1063/1.5063461

Submitted: 27 September 2018 . Accepted: 11 November 2018 . Published Online: 28 November 2018

Mohsin Habib (D), Murat Gokbayrak, Ekmel Ozbay, and Humeyra Caglayan
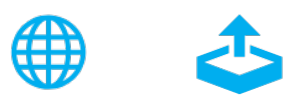

\section{ARTICLES YOU MAY BE INTERESTED IN}

Controllable generation of second-harmonic vortex beams through nonlinear supercell grating

Applied Physics Letters 113, 221101 (2018); https://doi.org/10.1063/1.5050423

Electrical tuning of metal-insulator-metal metasurface with electro-optic polymer

Applied Physics Letters 113, 231102 (2018); https://doi.org/10.1063/1.5054964

Integration of quantum dots with lithium niobate photonics

Applied Physics Letters 113, 221102 (2018); https://doi.org/10.1063/1.5054865

Lake Shore

CRYOTRONICS

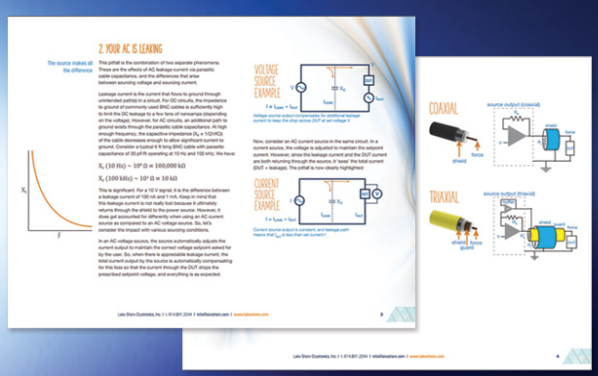

5 Electronic

- Measurement Pitfalls to Avoid

Get the whitepaper $\bullet$ 


\title{
Electrically controllable plasmon induced reflectance in hybrid metamaterials
}

\author{
Mohsin Habib, ${ }^{1,2,3}$ Murat Gokbayrak, ${ }^{2}$ Ekmel Ozbay, ${ }^{2,3,4,5}$ and Humeyra Caglayan ${ }^{1, a)}$ \\ ${ }^{1}$ Laboratory of Photonics, Tampere University, 33720 Tampere, Finland \\ ${ }^{2}$ Nanotechnology Research Center, Bilkent University, Ankara 06800, Turkey \\ ${ }^{3}$ Department of Electrical and Electronics Engineering, Bilkent University, Ankara 06800, Turkey \\ ${ }^{4}$ Department of Physics, Bilkent University, Ankara 06800, Turkey \\ ${ }^{5}$ UNAM-National Institute of Materials Science and Nanotechnology, Bilkent University, \\ Ankara 06800, Turkey
}

(Received 27 September 2018; accepted 11 November 2018; published online 28 November 2018)

\begin{abstract}
The tunable plasmon induced reflectance (PIR) effect has been numerically investigated and experimentally realized by hybrid metal-graphene metamaterials. The PIR effect is produced by two parallel strips of gold $(\mathrm{Au})$ and controlled electrically by applying the gate voltage to the graphene layer. The PIR response is generated by the weak hybridization of two bright modes of the gold strips and tuned by changing the Fermi level $\left(E_{f}\right)$ of the graphene. The total shift of $211.7 \mathrm{~nm}$ was achieved in the reflection peak by applying only $3 \mathrm{~V}$. This concept of real time electrical tuning of PIR, with a modulation depth of $\sim 49 \%$ and a spectral contrast ratio of $66.6 \%$, can be used for designing optical switches, optical modulators, and tunable sensors. Published by AIP Publishing. https://doi.org/10.1063/1.5063461
\end{abstract}

Electromagnetically induced transparency (EIT) is a phenomenon that can be produced by quantum interference between the two excitation pathways of a laser-activated atomic medium. ${ }^{1,2}$ This can be used for slow light applications, ${ }^{3}$ optical storage, ${ }^{4}$ optical switching, ${ }^{5}$ biosensing applications, ${ }^{6,7}$ and quantum information processing. ${ }^{8}$ Recently, plasmon induced transparency (PIT), analogous to the EITeffect, has been investigated in different platforms. The PIT has been observed in metallic nanoparticles, ${ }^{9,10}$ metamaterial structures, ${ }^{11-13}$ plasmonic coupled nanocavities, ${ }^{14}$ hybrid plasmon waveguide systems, ${ }^{15}$ and integrated photonic structures. ${ }^{16}$ Among these, metamaterial based structures are the best option as they have been a powerful tool to control the interaction of light with matter. ${ }^{17-22}$ A variety of tuning mechanisms are possible to generate the tunable response of metamaterials, ${ }^{23-26}$ which are electrical, ${ }^{27-29}$ thermal, ${ }^{30}$ or mechanical. ${ }^{31}$

To obtain PIT, the plasmonic mode can be either radiative (bright mode) or subradiant (dark mode). ${ }^{32}$ If the incident light couples directly, then it will produce bright modes that are spectrally broadened due to radiative damping. On the other hand, if the incident light is not coupled directly, it will generate dark modes that are weakly damped and spectrally narrow. ${ }^{33}$ PIT has been realized by either the destructive interference of dark-bright modes ${ }^{11}$ or detuning of the two bright modes. ${ }^{34}$

Hybrid metamaterial based devices can generate a tunable PIT response that can be used for enhanced sensing and switchable camouflage systems. ${ }^{29,35-37}$ These devices operate in the transmission mode and require a transparent substrate that limits the design wavelengths based on the type of substrate being used. ${ }^{11-13,34}$ On the other hand, the light matter interaction of graphene on top of the reflecting surface is

\footnotetext{
a) Author to whom correspondence should be addressed: humeyra.caglayan@ tut.fi
}

increased fourfold. ${ }^{38}$ This enhanced local electric field on a reflecting surface in the presence of graphene can be used for better detection and modulation. ${ }^{39-42}$ Therefore, a plasmon induced reflectance (PIR) will be more promising and can be used for efficient optoelectronic devices. The PIR-effect has only been experimentally realized by Chun-Feng et al. in 2014 . $^{43}$ The design is based on the cut-wire and split ring resonator acting as dark and bright modes, respectively. However, a dynamically tunable PIR design has not been investigated.

In this letter, we demonstrate a highly tunable PIR phenomenon with hybrid metal-graphene metamaterials. Our hybrid metamaterial based PIR device consists of a reflecting thin metallic film, a dielectric layer, and an electrically tunable hybrid metal-graphene structure, as shown in Fig. 1(a). a

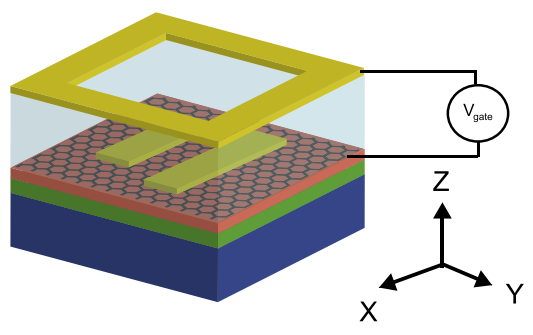

b

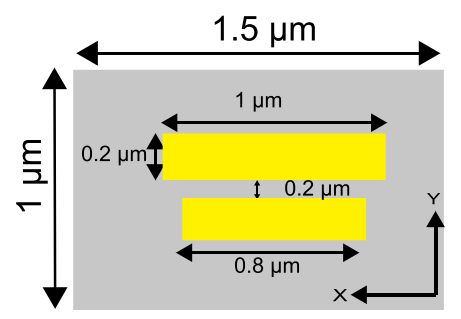

Gold strips

Graphene layer

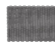

$\mathrm{Al}_{2} \mathrm{O}_{3}$

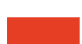

Al

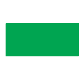

Si substrate

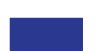

FIG. 1. Hybrid metamaterials for PIR. (a) 3D view of a PIR device with a schematic of the top gating. (b) The unit cell of a PIR device with two Au strips (golden). 
The two strips on top of the graphene layers will have their own plasmonic resonances, which can be controlled by the length of the strips, as shown in Fig. 1(b). The optical response of a PIR device can be engineered by two strips and the thickness of a dielectric layer. Moreover, this response can be broadly tunable by electrostatic doping. The optical properties of the graphene can be explained by the Pauli blocking principle. ${ }^{44}$ Two types of band transitions are possible depending upon the energy of the incident photon. If the energy of the photon is less than $2 E_{f}$, the intra-band transition is dominant. On the other hand, if the photon energy is higher than $2 E_{f}$, the inter-band transition is dominant. ${ }^{45,46}$ The interplay of these transitions establishes the optical response of graphene. The intra-band transitions in the conduction or valence band give rise to a Drude-like response similar to noble metals, and inter-band transitions that are Pauli blocked give rise to a universal, flat absorption spectrum. The Drude response is typically observed for frequencies up to the far-infrared, whereas the flat absorption of $2.3 \%$ is observed at optical frequencies. ${ }^{47}$

The surface conductivity of graphene can be modeled as an infinitesimally thin isotropic surface conductivity from both sides. The intra-band transition and inter-band transitions are presented in the following equations, respectively: ${ }^{48}$

$$
\begin{gathered}
\sigma_{\text {intra }}=-j \frac{e^{2} k_{B} T}{\pi \hbar^{2}(\omega-2 j \Gamma)}\left[\frac{\mu_{c}}{k_{B} T}+2 \ln \left(e^{\frac{-\mu_{c}}{k_{B} T}}+1\right)\right], \\
\sigma_{\text {inter }}=-j \frac{e^{2}}{4 \pi \hbar} \ln \left[\frac{2\left|\mu_{c}\right|-(\omega-2 j \Gamma) \hbar}{2\left|\mu_{c}\right|+(\omega-2 j \Gamma) \hbar}\right],
\end{gathered}
$$

where $e$ is the electron charge and $k_{B}$ is the Boltzmann constant. Other parameters include the temperature $(T)$, scattering rate $(\Gamma)$, angular frequency $\left(\omega_{c}\right)$, and chemical potential $\left(\mu_{c}\right)$ which can be changed in numerical investigations.

Overall, graphene has three major advantages. First, being a 2D material, it enables device miniaturization down to the atomic length scale. Second, the doping level in monolayer graphene is highly tunable by applying a voltage to an electrical gate. Third, graphene exhibits an optical response ranging from terahertz to optical frequencies, ${ }^{49}$ allowing for ultra-broadband operation. In particular, broadband optical modulators have been demonstrated. ${ }^{50}$

We have numerically investigated a PIR device using the finite difference time-domain (FDTD) method using Lumerical FDTD Solutions. The unit cell of the design has two parallel Au strips. Both strips serve as two bright modes. Weak hybridization of these bright modes results in the PIReffect. The lengths of strips are selected as $0.8 \mu \mathrm{m}$ and $1 \mu \mathrm{m}$ and the width as $\mathrm{W}=200 \mathrm{~nm}$ for both the strips, and the distance between two strips is kept as $\mathrm{D}=200 \mathrm{~nm}$. The periodic boundary condition is used for the $\mathrm{x}$ and $\mathrm{y}$ axes of the unit cell, and perfect matched layers (PMLs) are defined in the propagation direction. The design has a periodicity of $1.5 \mu \mathrm{m}$ in the $x$-axis and $1.0 \mu \mathrm{m}$ in the $y$-axis. A plane wave source along the $z$ direction was used to illuminate the unit cell having the electric field component $(E)$ parallel to the $x$-axis. For tuning the PIR response, four different values of $E_{f}$ for graphene were investigated. By changing the $E_{f}$ value which is equal to $\mu_{c}$, the intra-band transition is changed, and the optical response of the overall device is tailored. The scattering rate $(\Gamma)$ of graphene was set as $0.01 \mathrm{eV}\left(2.4 \times 10^{12} \mathrm{~s}^{-1}\right)$, and the temperature $(T)$ was set as $300 \mathrm{~K}$.

The device fabrication is done by cleaning the Si substrate using oxygen plasma to remove the residue of the photoresist used for dicing. $\mathrm{Al}$ is deposited using an E-beam evaporator, and $50 \mathrm{~nm}$ of $\mathrm{Al}_{2} \mathrm{O}_{3}$ is deposited using atomic layer deposition (ALD). ALD is a technique in which a chemical reaction takes place on the surface to form a $1 \mathrm{~nm}$ thick oxide layer in each step. The process results in highly uniform thin films because of the slow rate. We have transferred graphene on top of the oxide layer with the wet transfer method. Poly (methyl methacrylate) (PMMA) A4 resist was spin coated and baked, and the sample was coated with a conductive polymer like aquaSAVE. The sample is exposed to E-beam lithography. After the exposure, the resist is developed using a solution of methyl isobutyl ketone (MIBK):isopropyl alcohol (IPA) (1:1). Once the sample is patterned, $5 / 50 \mathrm{~nm} \mathrm{Ti} / \mathrm{Au}$ was coated on patterned samples. Both metals were deposited using an E-beam evaporator. Structures were visible after the lift-off process in acetone for $24 \mathrm{~h}$. The scanning electron microscopy (SEM) image of PIR structures is shown in the inset of Fig. 2(a).

In order to apply the gate voltage to graphene, $\mathrm{BaF}_{2}$ substrates with the metal contacts at the corners were placed on top of the PIR device and separated by an insulating tape. An
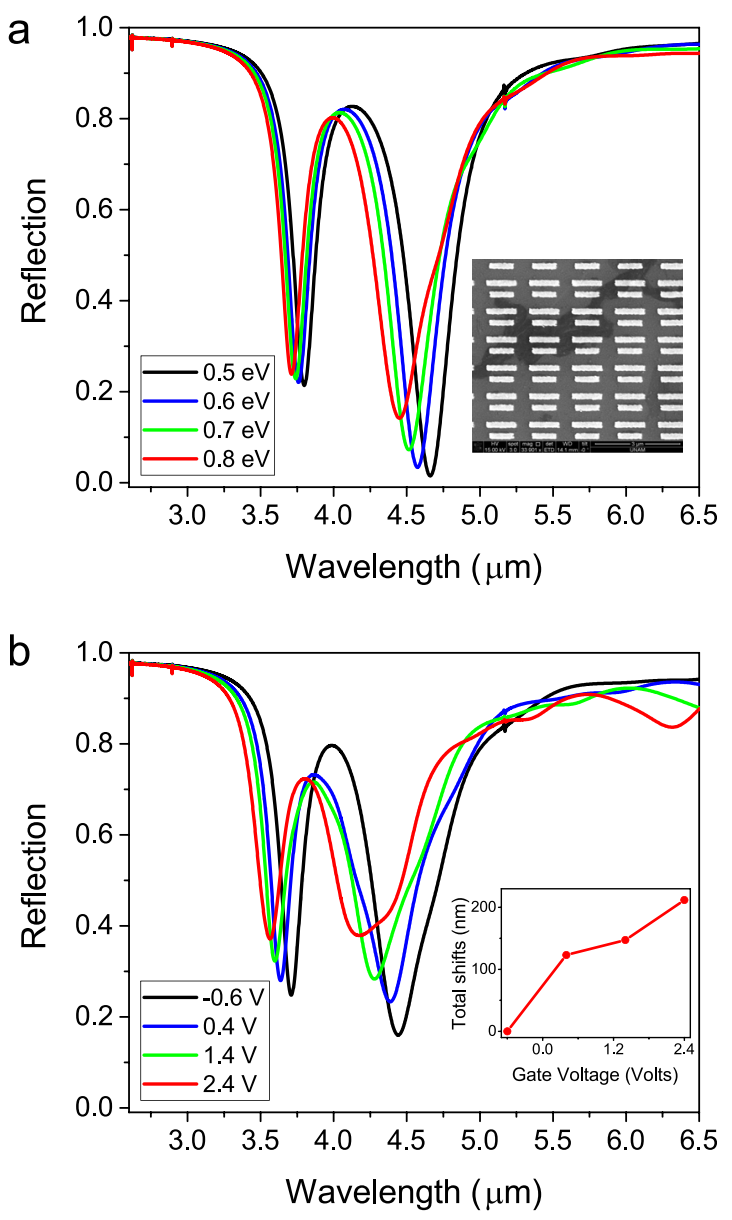

FIG. 2. Simulated and FTIR measurement of PIR structures. (a) Simulated results for different $E_{f}$ values. (b) Normalized reflection at $-0.6 \mathrm{~V}, 0.4 \mathrm{~V}$, $1.4 \mathrm{~V}$, and $2.4 \mathrm{~V}$. The total shift of the reflection peak and SEM image are presented in the inset. 
ionic liquid is inserted between the two substrates, and a source was connected to the graphene layer and metal contacts using conductive tape. By applying the gate voltage, the capacitance is produced between the graphene layer and the gold contact due to the presence of ionic liquid. ${ }^{25,26} \mathrm{~A}$ schematic of the top gating method is shown in Fig. 1(a). By controlling this induced field due to capacitance, we have controlled the carrier concentration accumulation near the surface of graphene. The $E_{f}$ of graphene can be controlled by the carrier concentration as discussed earlier.

The transfer of graphene from $\mathrm{Cu}$ to the substrate dopes graphene chemically and changes the $E_{f}$ slightly. In order to find this new value, the charge neutrality point (CNP) is measured. It is the point where the resistance has a maximum value and the capacitance has a minimum value for the applied voltage. It is measured to be $-0.6 \mathrm{~V}$, and the relative $E_{f}$ is $0.5 \mathrm{eV}$ for simulations. ${ }^{25}$

Reflection measurements for a PIR device was obtained by using a Fourier-transform infrared spectroscopy (FTIR) instrument integrated with a microscope. For the measurement, samples were illuminated with normal incidence. Measurements were done from 2.5 to $6.5 \mu \mathrm{m}$ at different gate voltages using the top gating. The $E_{f}$ of the graphene is changed from $0.5 \mathrm{eV}$ to $0.8 \mathrm{eV}$ for simulation in order to match the experimental curves obtained. These values are treated as fitting parameters as the $\mathrm{I}-\mathrm{V}$ measurement similar to previous works. ${ }^{25,26,29}$

The reflection simulation and experimental results are presented in Figs. 2(a) and 2(b), respectively. The PIR response is shifted toward shorter wavelengths as the $E_{f}$ of graphene is increased, as shown in Fig. 2(a). As the gate voltage is increased from $-0.6 \mathrm{~V}$ to $2.4 \mathrm{~V}$, a blueshift is observed in the PIR-effect, shown in Fig. 2(b). The total shift of $211.7 \mathrm{~nm}$ was observed by changing the gate voltage of $3.0 \mathrm{~V}$, presented in the inset of Fig. 2(b). The charge density in the graphene layer is increased by increasing the gate voltage from $-0.6 \mathrm{~V}$ to $2.4 \mathrm{~V}$. This will shift the resonance frequency of graphene, which is directly proportion to $E_{f}$. Therefore, the blueshift is observed by increasing $E_{f}$ in this design. In order to quantitatively describe the change in the reflection intensity with the change in the $E_{f}$ of graphene, the modulation depth in reflectance is defined as

$$
M_{\text {depth }}=\frac{\left|\left(R-R_{g}\right)\right|}{R} \times 100 .
$$

Here, $R$ is the reflection at $-0.6 \mathrm{~V}$ and $R_{g}$ is the reflection at $2.4 \mathrm{~V}$. The modulation depth $\left(M_{\text {depth }}\right)$ of $49 \%$ is realized at $4 \mu \mathrm{m}$.

The E-field investigation of two strips is made for three different wavelengths, as shown in Fig. 3. Electric field magnitudes are presented at two resonance wavelengths $(3.65 \mu \mathrm{m}$ and $4.42 \mu \mathrm{m}$ ) in Figs. 3(a) and 3(c). Moreover, the E-field magnitude is shown at the PIR wavelength $(3.87 \mu \mathrm{m})$ in Fig. 3 (b). These figures show that both strips are excited separately at the resonance wavelengths and serve as the bright mode resonances. On the other hand, at $3.87 \mu \mathrm{m}$, the PIR effect is produced by the simultaneous excitation of both strips.

The spectral contrast ratio $\left(S_{c o n}\right)$ for PIR is used to evaluate the performance of devices in sensing or optoelectronic applications, and it is described as
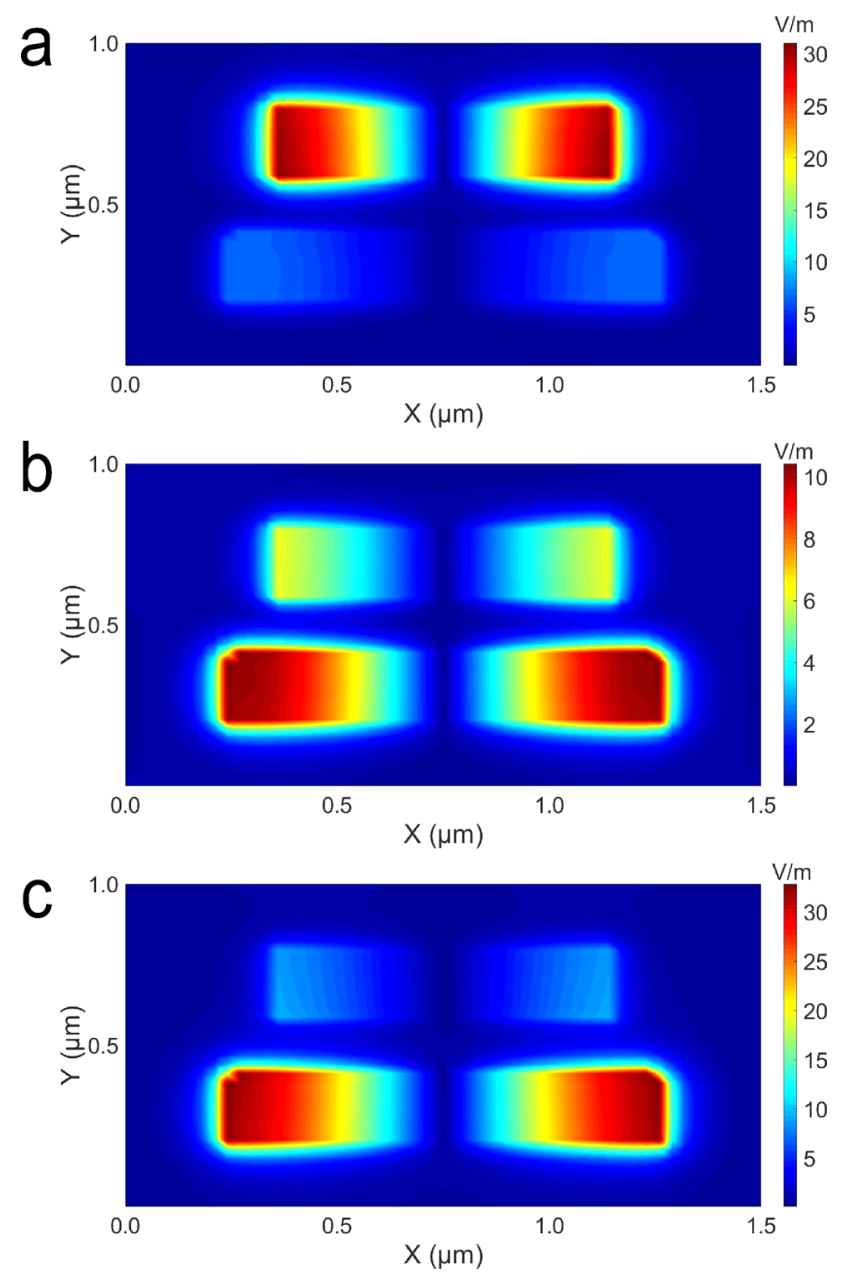

FIG. 3. E-field magnitude at $0.8 \mathrm{eV}$ : (a) E-field magnitude at $3.65 \mu \mathrm{m}$, (b) Efield magnitude at $3.87 \mu \mathrm{m}$, and (c) E-field magnitude at $4.42 \mu \mathrm{m}$.

$$
S_{\text {con }}=\frac{\left(R_{\text {peak }}-R_{\text {dip }}\right)}{\left(R_{\text {peak }}+R_{\text {dip }}\right)} \times 100,
$$

where $R_{\text {peak }}$ is the intensity of the reflection peak and $R_{d i p}$ is the intensity of the resonance dip. The $S_{c o n}$ of our design is $66.6 \%$. The PIR device is suitable for filtering and switching applications.

To summarize, PIR has been numerically investigated and experimentally realized using two strips on top of graphene. By changing the $E_{f}$ of graphene, the PIR response was shifted. A large tuning range was demonstrated for the FTIR measurements of PIR structures by applying gate voltage. We were able to obtain a $211.7 \mathrm{~nm}$ shift in the reflection window and a modulation depth of $49 \%$ by applying gate voltage up to $3 \mathrm{~V}$. These results of PIR can be used in many fields such as slow light applications and nonlinear optics.

This work was supported by TUBITAK under Project No. 117F150. Competitive funding to strengthen university research profiles was funded by the Academy of Finland, Decision No. 301820. One of the authors (E.O.) also acknowledges the partial support from the Turkish Academy of Sciences.

${ }^{1}$ K.-J. Boller, A. Imamoğlu, and S. E. Harris, "Observation of electromagnetically induced transparency," Phys. Rev. Lett. 66, 2593 (1991).

${ }^{2}$ J. P. Marangos, "Electromagnetically induced transparency," J. Mod. Opt. 45, 471-503 (1998). 
${ }^{3}$ T. F. Krauss, "Why do we need slow light?," Nat. Photonics 2, 448 (2008). ${ }^{4}$ I. Novikova, R. L. Walsworth, and Y. Xiao, "Electromagnetically induced transparency-based slow and stored light in warm atoms," Laser Photonics Rev. 6, 333-353 (2012).

${ }^{5}$ J. Chen, P. Wang, C. Chen, Y. Lu, H. Ming, and Q. Zhan, "Plasmonic EIT-like switching in bright-dark-bright plasmon resonators," Opt. Express 19, 5970-5978 (2011).

${ }^{6}$ Z. Vafapour, Y. Hajati, M. Hajati, and H. Ghahraloud, "Graphene-based mid-infrared biosensor," JOSA B 34, 2586-2592 (2017).

${ }^{7} \mathrm{Z}$. Vafapour, "Near infrared biosensor based on classical electromagnetically induced reflectance (CL-EIR) in a planar complementary metamaterial," Opt. Commun. 387, 1-11 (2017).

${ }^{8}$ M. Fleischhauer, A. Imamoglu, and J. P. Marangos, "Electromagnetically induced transparency: Optics in coherent media," Rev. Mod. Phys. 77, 633 (2005).

${ }^{9}$ C. Rohde, K. Hasegawa, and M. Deutsch, "Plasmon-assisted transparency in metal-dielectric microspheres," Opt. Lett. 32, 415-417 (2007).

${ }^{10}$ V. Yannopapas, E. Paspalakis, and N. V. Vitanov, "Electromagnetically induced transparency and slow light in an array of metallic nanoparticles," Phys. Rev. B 80, 035104 (2009).

${ }^{11}$ N. Liu, L. Langguth, T. Weiss, J. Kästel, M. Fleischhauer, T. Pfau, and H. Giessen, "Plasmonic analogue of electromagnetically induced transparency at the Drude damping limit," Nat. Mater. 8, 758 (2009).

${ }^{12}$ Y. Zhu, X. Hu, Y. Fu, H. Yang, and Q. Gong, "Ultralow-power and ultrafast all-optical tunable plasmon-induced transparency in metamaterials at optical communication range," Sci. Rep. 3, 2338 (2013).

${ }^{13}$ M. P. Hokmabadi, E. Philip, E. Rivera, P. Kung, and S. M. Kim, "Plasmon-induced transparency by hybridizing concentric-twisted double split ring resonators," Sci. Rep. 5, 15735 (2015).

${ }^{14}$ Y. Zhu, X. Hu, H. Yang, and Q. Gong, "On-chip plasmon-induced transparency based on plasmonic coupled nanocavities," Sci. Rep. 4, 3752 (2014).

${ }^{15}$ J. Zhang, W. Bai, L. Cai, Y. Xu, G. Song, and Q. Gan, "Observation of ultra-narrow band plasmon induced transparency based on large-area hybrid plasmon-waveguide systems," Appl. Phys. Lett. 99, 181120 (2011). ${ }^{16}$ Y. A. Vlasov, M. O'boyle, H. F. Hamann, and S. J. McNab, "Active control of slow light on a chip with photonic crystal waveguides," Nature 438 , 65 (2005).

${ }^{17}$ Y. Fan, N.-H. Shen, T. Koschny, and C. M. Soukoulis, "Tunable terahertz meta-surface with graphene cut-wires," ACS Photonics 2, 151-156 (2015).

${ }^{18}$ Y. Fan, N.-H. Shen, F. Zhang, Q. Zhao, Z. Wei, P. Zhang, J. Dong, Q. Fu, H. Li, and C. M. Soukoulis, "Photoexcited graphene metasurfaces: Significantly enhanced and tunable magnetic resonances," ACS Photonics 5, 1612-1618 (2018).

${ }^{19}$ H. Tao, W. J. Padilla, X. Zhang, and R. D. Averitt, "Recent progress in electromagnetic metamaterial devices for terahertz applications," IEEE J. Sel. Top. Quantum Electron. 17, 92-101 (2011).

${ }^{20}$ J. B. Pendry, D. Schurig, and D. R. Smith, "Controlling electromagnetic fields," Science 312, 1780-1782 (2006).

${ }^{21}$ E. Ozbay, "Plasmonics: Merging photonics and electronics at nanoscale dimensions," Science 311, 189-193 (2006).

${ }^{22}$ O. Balci, N. Kakenov, E. Karademir, S. Balci, S. Cakmakyapan, E. O. Polat, H. Caglayan, E. Özbay, and C. Kocabas, "Electrically switchable metadevices via graphene," Sci. Adv. 4, eaao1749 (2018).

${ }^{23}$ T.-T. Kim, H.-D. Kim, R. Zhao, S. S. Oh, T. Ha, D. S. Chung, Y. H. Lee, B. Min, and S. Zhang, "Electrically tunable slow light using graphene metamaterials," ACS Photonics 5, 1800-1807 (2018).

${ }^{24}$ S. Cakmakyapan, H. Caglayan, and E. Ozbay, "Coupling enhancement of split ring resonators on graphene," Carbon 80, 351-355 (2014).

${ }^{25}$ O. Ozdemir, A. M. Aygar, O. Balci, C. Kocabas, H. Caglayan, and E. Ozbay, "Enhanced tunability of V-shaped plasmonic structures using ionic liquid gating and graphene," Carbon 108, 515-520 (2016).

${ }^{26}$ A. M. Aygar, O. Balci, S. Cakmakyapan, C. Kocabas, H. Caglayan, and E. Ozbay, "Comparison of back and top gating schemes with tunable graphene fractal metasurfaces," ACS Photonics 3, 2303-2307 (2016).

${ }^{27}$ M. Goldflam, T. Driscoll, B. Chapler, O. Khatib, N. Marie Jokerst, S. Palit, D. Smith, B.-J. Kim, G. Seo, H.-T. Kim et al., "Reconfigurable gradient index using $\mathrm{VO}_{2}$ memory metamaterials," Appl. Phys. Lett. 99, 044103 (2011).

${ }^{28}$ Y. Urzhumov, J. S. Lee, T. Tyler, S. Dhar, V. Nguyen, N. M. Jokerst, P. Schmalenberg, and D. R. Smith, "Electronically reconfigurable metal-onsilicon metamaterial," Phys. Rev. B 86, 075112 (2012).

${ }^{29}$ M. Habib, A. R. Rashed, E. Ozbay, and H. Caglayan, "Graphene-based tunable plasmon induced transparency in gold strips," Opt. Mater. Express 8, 1069-1074 (2018).

${ }^{30}$ N. I. Zheludev and Y. S. Kivshar, "From metamaterials to metadevices," Nat. Mater. 11, 917 (2012).

${ }^{31}$ D. Shin, Y. Urzhumov, Y. Jung, G. Kang, S. Baek, M. Choi, H. Park, K. Kim, and D. R. Smith, "Broadband electromagnetic cloaking with smart metamaterials," Nat. Commun. 3, 1213 (2012).

${ }^{32}$ M. I. Stockman, S. V. Faleev, and D. J. Bergman, "Localization versus delocalization of surface plasmons in nanosystems: Can one state have both characteristics?," Phys. Rev. Lett. 87, 167401 (2001).

${ }^{33}$ A. E. Miroshnichenko, S. Flach, and Y. S. Kivshar, "Fano resonances in nanoscale structures," Rev. Mod. Phys. 82, 2257 (2010).

${ }^{34}$ H. Zhang, Y. Cao, Y. Liu, Y. Li, and Y. Zhang, "A novel graphene metamaterial design for tunable terahertz plasmon induced transparency by two bright mode coupling," Opt. Commun. 391, 9-15 (2017).

${ }^{35}$ F. Xiao, W. Zhu, W. Shang, T. Mei, M. Premaratne, and J. Zhao, "Electrical control of second harmonic generation in a graphene-based plasmonic Fano structure," Opt. Express 23, 3236-3244 (2015).

${ }^{36}$ C. Liu, P. Liu, C. Yang, Y. Lin, and H. Liu, "Analogue of dual-controlled electromagnetically induced transparency based on graphene metamaterial," Carbon 142, 354-362 (2018).

${ }^{37}$ X. He, X. Yang, G. Lu, W. Yang, F. Wu, Z. Yu, and J. Jiang, "Implementation of selective controlling electromagnetically induced transparency in terahertz graphene metamaterial," Carbon 123, 668-675 (2017).

${ }^{38}$ Y. Liu, Y. Dai, Q. Feng, Y. Shan, L. Du, Y. Xia, G. Lu, F. Liu, G. Du, C. Tian et al., "Enhanced light-matter interactions in graphene-covered dielectric magnetic mirrors," Opt. Express 25, 30754-30763 (2017).

${ }^{39}$ L. Gao, W. Ren, B. Liu, R. Saito, Z.-S. Wu, S. Li, C. Jiang, F. Li, and H.-M. Cheng, "Surface and interference coenhanced Raman scattering of graphene," ACS Nano 3, 933-939 (2009).

${ }^{40}$ Z. Fang, Z. Liu, Y. Wang, P. M. Ajayan, P. Nordlander, and N. J. Halas, "Graphene-antenna sandwich photodetector," Nano Lett. 12, 3808-3813 (2012).

${ }^{41}$ F. Xia, T. Mueller, Y.-M. Lin, A. Valdes-Garcia, and P. Avouris, "Ultrafast graphene photodetector," Nat. Nanotechnol. 4, 839 (2009).

${ }^{42}$ M. Furchi, A. Urich, A. Pospischil, G. Lilley, K. Unterrainer, H. Detz, P. Klang, A. M. Andrews, W. Schrenk, G. Strasser et al., "Microcavity-integrated graphene photodetector," Nano Lett. 12, 2773-2777 (2012).

${ }^{43}$ D. Chun-Feng, Z. Ya-Ting, Y. Jian-Quan, S. Chong-Ling, X. De-Gang, and Z. Gui-Zhong, "Reflection-type electromagnetically induced transparency analogue in terahertz metamaterials," Chin. Phys. B 23, 124203 (2014).

${ }^{44}$ K. F. Mak, L. Ju, F. Wang, and T. F. Heinz, "Optical spectroscopy of graphene: From the far infrared to the ultraviolet," Solid State Commun. 152, 1341-1349 (2012).

${ }^{45}$ V. Gusynin, S. Sharapov, and J. Carbotte, "Unusual microwave response of Dirac quasiparticles in graphene," Phys. Rev. Lett. 96, 256802 (2006).

${ }^{46}$ O. Balci, E. O. Polat, N. Kakenov, and C. Kocabas, "Graphene-enabled electrically switchable radar-absorbing surfaces," Nat. Commun. 6, 6628 (2015).

${ }^{47}$ Q. Bao and K. P. Loh, "Graphene photonics, plasmonics, and broadband optoelectronic devices," ACS Nano 6, 3677-3694 (2012).

${ }^{48}$ G. W. Hanson, "Dyadic green's functions and guided surface waves for a surface conductivity model of graphene," J. Appl. Phys. 103, 064302 (2008).

${ }^{49}$ W. Zhu, F. Xiao, M. Kang, D. Sikdar, and M. Premaratne, "Tunable terahertz left-handed metamaterial based on multi-layer graphene-dielectric composite," Appl. Phys. Lett. 104, 051902 (2014).

${ }^{50}$ F. Wang, Y. Zhang, C. Tian, C. Girit, A. Zettl, M. Crommie, and Y. R. Shen, "Gate-variable optical transitions in graphene," Science 320, 206-209 (2008). 